Instituto Internacional de Investigación y Desarrollo Tecnológico Educativo INDTEC, C.A.

DOI: https://doi.org/10.29394/Scientific.issn.2542-2987.2018.3.10.13.248-266

OAI-PMH: http://www.indteca.com/ojs/index.php/Revista Scientific/oai

Ensayo Original / Original Essay

\title{
Enfoque Complejo en la Gestión del Conocimiento en Investigación
}

Autora: Ingrid Yorley Guerrero Rivera Universidad Fermín Toro, UFT teacher272777@gmail.com

Mérida, Venezuela

\begin{abstract}
Resumen
El presente ensayo precisa diversos planteamientos teóricos desde aportes de Morín, Osorio, otros; los cuales permiten reflexionar sobre la efectividad de la gestión del conocimiento en investigación dentro de la Universidad Politécnica Territorial Manuela Sáenz (UPTMS), todo esto desde un enfoque complejo con sentido de pertinencia a la realidad social actual, dado que el conocimiento es un recurso muy apreciable para las instituciones de Educación Universitaria. Por cuanto, en la Universidad Politécnica Territorial Manuela Sáenz contar con talento humano altamente calificado es fundamental; pero el reto indiscutible es innovar para aumentar y mejorar la capacidad gerencial en investigación, con la finalidad de administrar correctamente el conocimiento que es generado internamente en esta institución desde un enfoque complejo.
\end{abstract}

Palabras clave: gestión del conocimiento; investigación; organización. 


\section{Complex Approach in Knowledge Management in Research}

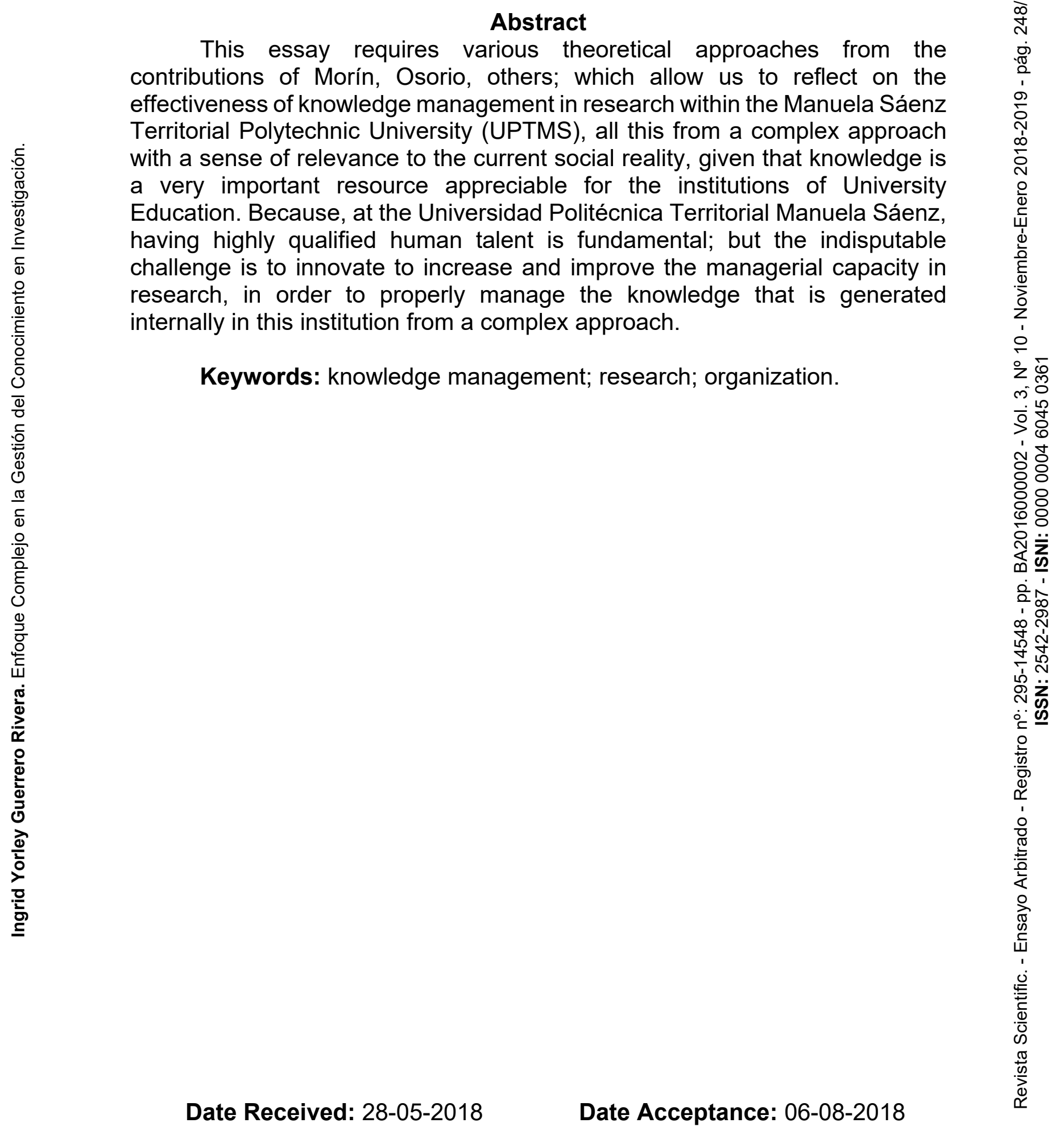




\section{Planteamiento Introductorio}

En los últimos años, se ha asumido que la fuente de la ventaja competitiva se aloja en la capacidad de las organizaciones para adquirir, transmitir, y aplicar conocimiento. La praxis gerencial requiere de grandes actualizaciones e innovaciones que según investigaciones de autores declaran, que es un período de cambios y de la era planetaria, por lo que se avecina una etapa con énfasis en el conocimiento y la creatividad para el progreso y desarrollo de toda la institución. Barroso (2011): puntualiza: “...en la actualidad el conocimiento se ha situado como el primordial capital que poseen las personas, las instituciones, organizaciones y naciones para manejarse en el nuevo orden mundial" (pág. 3).

Según esta postura, se considera que el conocimiento es imprescindible para mejorar continuamente los procesos organizacionales, por tanto, se emplea para producir innovación, la generación del conocimiento pretende ampliar y profundizar el rendimiento de lo que ya se conoce, puesto que el conocimiento sea cual sea el área de donde provenga debe ser instrumento para conseguir nuevas e innovadoras ideas en beneficio toda organización, en términos de epistemología, se diría que es preciso determinar las intersubjetividades en donde la productividad del conocimiento va a ser cada día más, el factor crucial en la posición competitiva de toda Institución.

A tal efecto, el conocimiento es hoy el insumo primordial que inspira a las instituciones universitarias a mantenerse en constante innovación, alimentando así, su crecimiento y desarrollo académico. Este insumo se encuentra en cada uno de los miembros de la institución, y en la medida que las universidades sean capaces de direccionarlo correctamente, lograrán contar y disponer de la fundamental e importante ventaja competitiva de la nueva era planetaria, destacándose así como universidades de renombre académico y organizaciones inteligentes.

Para León, Tejada y Yataco (2003): La organización inteligente es 
aquella que trata constantemente de asegurar que los miembros quienes conforman la organización aprendan y exploten todo su potencial y capacidades y habilidades; "esto es la capacidad de comprender la complejidad de adquirir compromisos, de asumir su responsabilidad, de buscar el continuo auto-crecimiento, de crear sinergias a través del trabajo en equipo, sostenibilidad productiva académica y de transferencia de conocimientos" (pág. 82).

Por otra parte, los cimientos de la sociedad del conocimiento en las universidades, se rigen por la innovación y capacidad intelectual de sus miembros, las tecnologías de información y comunicación (TIC), así como por la motivación que tienen para relacionarse social, económica y culturalmente en el seno de la comunidad universitaria.

Por ende, es oportuno destacar lo relevante que es la gestión del conocimiento en investigación dentro de la Universidad Politécnica Territorial Manuela Sáenz. Dado que, como cualquier universidad no se escapa de la realidad actual en la que se encuentran todas, como son los invariables cambios tecnológicos que influyen en ellas; ocasionando efectos no deseados. Pero esto no siempre deja de ser alentador, ya que se busca constantemente, que sean organizaciones inteligentes, con conocimientos nuevos e innovadores, a fin de dar respuesta al compromiso social que tienen dentro de un contexto complejo.

Es por esto que, la gestión del conocimiento (GC) dentro de las instituciones universitarias, permite aprovechar al máximo los recursos existentes para que las personas desarrollen su potencial intelectual sobre un tema específico.

La gestión del conocimiento desde el contexto ontológico, en investigación, visto desde la complejidad, involucra un conjunto de procesos gerenciales, con la finalidad de generar nuevos conocimientos acercándose a una verdad que le dé sentido y respuesta a la realidad que encierra el contexto 
universitario.

En consecuencia, el presente ensayo procura dilucidar si efectivamente la gestión del conocimiento en investigación desde el enfoque complejo, tiene capacidad explicativa sobre la eficacia institucional. La gestión del conocimiento en investigación en la Universidad Politécnica Territorial Manuela Sáenz (UPTMS), constituye un tema fundamental, como sistema organizacional. Así, partiendo de diversos planteamientos teóricos a lo largo del presente análisis, se busca avanzar hacia la efectividad en la gestión del conocimiento, lo cual facilite la adquisición, asociación, e incorporación de nuevos saberes, así como formas científico-tecnológicas en las funciones gerenciales dentro de esta institución universitaria, con el objetivo de incrementar y mejorar sus niveles en las investigaciones; de manera que sea una organización más productiva.

Destacando que, la Universidad Politécnica Territorial Manuela Sáenz ha sido creada con el fin de municipalizar la educación y de impulsar la evolución de la educación universitaria en Venezuela; esta Institución como ente que agrupa una comunidad en general, posee la gran tarea de orientar hacia la búsqueda de la verdad, afianzar los valores y principios trascendentales del individuo, como derecho primordial apegado a la Constitución de nuestro país.

Por tanto, esta universidad emerge en el seno de la Misión Alma Mater, (Gaceta Oficial № 5.987, 2010):

Con carácter social humanístico, con la labor de ahondar, fortalecer y promover un deseo añorado, nacido de las labores, aspiraciones y de la fuerza creativa de los habitantes de García de Hevia y otros Municipios de la zona Norte del estado, la cual, se consideró no solo como una necesidad, sino además potencialidad, y fue conscientemente encaminado por medio de las jurisdicciones regionales y la Fundación para el Desarrollo, Tecnológico, Educativo y Científico (núm. 39468). 


\section{Desarrollo}

\subsection{La Complejidad como Enfoque Integrador en la Gestión del Conocimiento en Investigación}

Hoy las universidades sufren las consecuencias de los grandes desajustes y la situación problemática que atraviesa el país, es incuestionable que los problemas sociopolíticos, económicos y culturales están afectando las Universidades Venezolanas, puesto que sería un error no asumirlo, ya que, la universidad es parte de la sociedad y no escapa de todo esto. Del mismo modo sucede con la investigación, la cual es parte esencial de esta; y por ende debe existir un inseparable vínculo entre universidad-conocimiento-investigación y sociedad, términos que lamentablemente se encuentran en una total desarticulación y divorcio; es decir van por caminos bifurcados.

De ahí, Ramos e Isa (2010), exponen: "ciertamente el contexto social, político económico y cultural que rodea las universidades, hoy día ejerce una influencia que no puede aislarse del conocimiento que dentro de ellas se produce, ni mucho menos, verse como sistemas aislados independientes e inconexos" (párr. 8).

De modo que, se debe encausar la universidad hacia la vigorosa tarea que siempre ha tenido encomendada, como es la búsqueda del desarrollo y la calidad social; pero, esto solo se puede lograr a través de la investigación universitaria; de modo que, hay que reconstruir y reorganizar las estructuras gerenciales de investigación dentro de la (UPTMS) buscando establecer políticas que no permitan fragmentar el conocimiento, las investigaciones y faciliten la constante revisión y mejora de anteriores investigaciones, utilizando el producto intelectual final de un investigador como punto de partida de otro, garantizando una dinámica de retroalimentación conveniente para avanzar de forma sostenida en una realidad cambiante.

Es decir, la complejidad como elemento integrador en la gestión del conocimiento en investigación en la (UPTMS), debe analizarse como el 
desarrollo y aplicación de un nuevo marco conceptual integrador, motivador y compartido por los miembros involucrados desde diversas disciplinas. De ahí que Gil, Domínguez, Garcia, Gándara y Mathison (2012), destacan que:

...la investigación debe tener la agilidad suficiente, para poder migrar entre una investigación básica de generación propia del saber y la otra investigación que es inmediata, que busca cómo resolver problemas en todos los ámbitos de la sociedad... para lograr tal propósito, la universidad tiene como reto el empezar a innovarse al interior. Este llamado ha llevado muy especialmente a las universidades a constituirse en organizaciones capaces de analizarse, cuestionarse y transformarse a la luz de lograr una mejor comprensión y transformación de los problemas educacionales en concordancia a los nuevos retos de formación (pág. 43).

Esto conlleva a que las universidades se adaptan a los cambios tecnológicos, entre otros, para obtener su meta y cumplir con lo establecido en la Ley de Universidades. Asimismo, García (2016), sostiene que:

...el papel de la universidad debe ser visto dentro de un sistema de educación superior, como el centro de una red que interconecta todo el conocimiento... una sociedad basada en el conocimiento, el tratamiento, almacenamiento, intercambio y producción de conocimiento prevalece, y la universidad, desde la perspectiva de su principal misión en cuanto a la producción, trasmisión y transferencia de conocimientos, puede y debe contribuir en la configuración de esa sociedad, basada en el conocimiento, transformándose y evolucionando hacia un modelo de docencia-investigación donde se gestione el conocimiento orientado a fortalecer un sistema efectivo, sistemático y coherente de interacción entre la universidad y su entorno (pág. 1).

Por su parte, la gerencia universitaria a través de los tiempos debe evolucionar desde diferentes disciplinas, teorías y paradigmas en concordancia con los cambios paradigmáticos y la evolución tecnológica, por estar indubitablemente orientada a su principal objetivo que es satisfacer las exigencias sociales; lo cual será posible a través de sus procesos 
investigativos; a fin de conseguir una verdadera transformación y reconstrucción universitaria.

Desde esta perspectiva la complejidad invita a un pensamiento reestructurador unificador capaz de unir y enlazar disciplinas a fin de generar conocimientos útiles, valiosos para la organización y por ende para la sociedad, es decir, conocimientos sin barreras ya que como mencionaba Morin cada disciplina separada momentáneamente permite profundizar en un conocimiento específico, pero luego al pasar el tiempo comienzan a mostrarse en un oscuralismo a lo que llamo racionalidad restringida, lo cual nos aparta de la complejidad organizada, y de una nueva visión de la realidad.

Dentro de este orden de ideas, gestionar el conocimiento en investigación, en las instituciones universitarias, se muestra cómo saber cuándo es considerado como un bien en sí mismo, esto conlleva que la generación del conocimiento convertido en ciencia se vuelve útil cuando trae consigo un beneficio a la sociedad, al mejoramiento y desarrollo de la misma, esto como función creadora de nuevas e innovadoras ideas.

De forma que se comprende que, dentro de los procesos investigativos los representantes se deben a una comunidad académica, Marín (2010): señala "...debe estar regida por un sistema cultural de la investigación, determinado por sus tramas de significados, conocimientos específicos y práctica, desde la que se tiene experiencia del mundo y se lo comprende, para ir tras él y transformarlo" (pág. 5).

De manera que, la gestión del conocimiento en investigación, implica una práctica que posibilita a los integrantes la configuración de modernas y novedosas formas de pensar, actuar y relacionarse con los que interactúan (investigadores). Este sistema incluye estructuras conceptuales, métodos, equipos de trabajo mecanismos de medición y grupos de personas que reconozcan además el trabajo en colectivo.

Ahora bien, el proceso de gestión del conocimiento en investigación en 
la Universidad Politécnica Territorial Manuela Sáenz, desde la complejidad, no es perceptible, y es muy difícil aceptar la realidad que enfrenta esta institución, ya que ciertamente hay una serie de cargos destinados a la producción de conocimientos para su puesta en práctica, pero la cuestión es que la universidad aún no ha asumido la innegable necesidad de generar conocimientos y saberes integrados, para mantenerse activa y así confrontar un mundo que cambia de una forma precipitada; Así como, proporcionar soluciones efectivas a los problemas diarios de la sociedad.

No obstante, la poca generación del conocimiento dentro de la universidad no se corresponde al propósito para la cual fueron creadas tales Instituciones.

En este contexto, en la mencionada universidad se generan conocimientos fragmentados, cada investigador es individualista; es decir, se producen conocimientos aislados, por cuanto debe alinearse este pensamiento hacia la integración de saberes tal como lo plantea la complejidad, donde emerjan nuevas ideas desde diversas disciplinas para generar de esta manera investigaciones novedosas, con el propósito de reorientar la organización a su crecimiento, desarrollo y al éxito.

Por lo tanto, es vital que todo gerente sea transformador, participativo que comprenda y se apropie de este pensamiento complejo, para superar las impredecibles realidades complejas.

En este sentido, estas organizaciones exigen líderes capaces de realizar transformaciones en su entorno. Al respecto, Fernández y Quintero (2017), aseveran que: "estos líderes transformacionales logran responder de manera rápida a las exigencias del entorno apoyados en sus seguidores, en quienes han influido, logrado el cambio de un interés individual a un interés colectivo, alcanzando las metas de la organización" (pág. 60).

Se confirma entonces que el tipo de liderazgo dentro de las instituciones es determinante para romper paradigmas y generar cambios significativos. De 
tal manera que en los equipos reales de investigación es fundamental el liderazgo transformacional, el cual rompa con el individualismo, y fomente a la exteriorización del conocimiento por parte de los investigadores científicos; igualmente, motive al equipo a buscar metas comunes, este líder debe poseer una actitud proactiva, positiva, holista orientada a la productividad y consolidación de espacios apropiados para intercambiar conocimientos.

Adicionalmente, el pensamiento complejo procura que broten nuevas formas de pensamiento, estrategias y acciones en el ser humano que lo reoriente en su forma de ver, entender el mundo y por ende enfrentarlo, parafraseando a Pereira (2010):

La función de un pensamiento complejo es develar la naturaleza y complejidad de todos los fenómenos y sus interrelaciones, esta tarea, debe estar implícita como compromiso para con nuestro mundo. Estamos inmersos en una época planetaria; por lo tanto, se debe enlazar la enseñanza de la especie humana con la ética del entendimiento entre los individuos. Las profundas reformas de pensamiento deben indiscutiblemente estar puestas al servicio de la humanidad, motivando a la comprensión, moral, solidaridad de los ciudadanos superando el individualismo y la autodestrucción (pág. 74).

Ontológicamente, se asume que el universo, la naturaleza es el resultado de las relaciones e interconexiones con los estímulos naturales y colectivos. Esta configuración filosófica, permite considerar que el saber humano no es adoptado de manera pasiva, de lo contrario se procesa y crea activamente. Para la teoría constructivista la objetividad en su totalidad aislada. bifurcada del individuo, no ostenta sentido alguno, puesto que el conocimiento se traduce como una exégesis, una reconstrucción y producción mental, resultando inconcebible apartar al investigador de lo que es investigado.

Por tanto, para Pérez y Alfonso (2016): "lo complejo es complexus 
porque se trata de acercamiento a múltiples planos, niveles e implicaciones que van configurando en el pensamiento la proyección de una explicación que se va articulando en los procesos de investigación" (pág. 15).

Según lo expuesto con anterioridad, es importante, dentro de la (UPTMS) incluir y promover el pensamiento complejo en la gestión del conocimiento en investigación, puesto que constituyen una estrategia eficaz, transformadora para su evolución, Balza (2009), señala que:

Se propugna que no es posible pretender investigar para comprender e interpretar realidades complejas con las viejas estructuras de un pensamiento simplificador y desintegrador, con los agotados posicionamientos epistemológicos fragmentados y parcelados de producir conocimientos, por lo tanto, es necesario asumir la complejidad como un modo de pensar para poder sentir la vida a plenitud (pág. 47).

Es por esto, que el proceso de investigar desde la complejidad debe coadyuvar en la solución de problemas de la sociedad llevando un control, armonizando la libertad de crear, innovar enseñar y aprender; a fin de evolucionar en el pensamiento, haciendo de este una herramienta capaz de generar nuevos pensamientos que conlleven a una máxima satisfacción en la calidad de vida de los ciudadanos.

En consecuencia, es un nuevo desafío a través de la investigación la renovación y reestructuración del conocimiento sin ruptura disciplinar, puesto que, parafraseando a Osorio (2012a):

resalta que nuestro mundo planetario se destaca por ser un mundo complicado, es decir en red, lo describe como "enredado" por un sin número de interrelaciones, donde los fenómenos cuales sean, de índole natural o social dependen entre sí, estos se analizan partiendo de su funcionamiento y ubicación con relación al todo del que son parte. Las funciones aisladas no existen, la posibilidad de llegar a un acuerdo fragmentado de nuestro entorno es nula. Por ende, para entender este mundo complejo, es preciso una nueva visión de la racionalidad humana; distinta a la que nos ofrecieron 
concepciones lógicas clásicas (pág. 281).

Sin embargo, esta vertiente de nueva racionalidad, debe llevar a una reforma y reestructuración sin precedentes en las instituciones universitarias, que coexisten actualmente con conocimientos generados en siglos pasados, cuando se creaban desde la estructura disciplinar de los saberes, sapiencias necesarias para otros tiempos, pero notablemente, desfasados con la realidad actual.

Por ende, la importancia del pensamiento complejo el cual, Osorio (2012b): "nos invita y reta para pensar religando, juntando, contextualizando; nos insta a ver e integrar los antagonismos y a desarrollar una visión poliocular de la realidad" (pág. 276).

De modo que, es tarea primordial de todos los gerentes de las instituciones fomentar el desarrollo de actividades e investigaciones presididas por un enfoque complejo, donde exista un discernimiento creativo, innovador, que responda a las verdaderas necesidades colectivas; que fortifiquen además las diversas practicas académico administrativas dentro de la institución.

Por otra parte, el sector universitario es el máximo delegado y/o actor en lo que concierne a la generación de conocimientos, por ello el valor que poseen las capacidades mentales calificadas dentro de las instituciones, se ha vuelto un tema de gran transcendencia. Por lo que, al hacer referencia a la generación del conocimiento, Berrio, Angulo y Gil (2013a), señalan: "que generar conocimientos es la actividad sustantiva de los centros de investigaciones científica; así también, complementariamente, apropiarse de él, validarlo, documentarlo; divulgarlo y/o propiciar su transferencia para el desarrollo de aplicaciones de beneficio social..." (pág. 123).

Es evidente, que las universidades y los Centros de investigación científica como principales comisionados, deben poseer una constante interrelación e integración en pro del beneficio social, con el fin de exteriorizar, 
propagar los conocimientos y desarrollar investigaciones de orden productivo para el país. Asimismo, Berrio, Angulo y Gil (2013b), acotan:

Es significativo, el interés de conocer como desde las universidades y centros de investigación se genera valor al conocimiento, su gestión es una forma de responder a problemáticas del entorno apoyado en la contribución de personas e instituciones y la responsabilidad de los equipos de trabajo. Estas consideraciones presuponen que no debe contemplarse la gestión del conocimiento como un simple proceso más en la institución, sino que necesita impulsar integramente las ventajas que proporciona la integración de la gestión del conocimiento en la estrategia organizacional. (pág. 117).

Ante esta exegesis, es imprescindible que desde la gerencia investigativa dentro de las instituciones de educación universitaria, se fomente estudios que tengan correspondencia con la amplia problemática que atraviesa el sector universitario y el país en general, asimismo crear las condiciones y escenarios para que se pueda desarrollar una cultura investigativa orientada a un pensamiento holístico y transdisciplinario enrumbado a producir un verdadero impacto en los contextos sociales y educativos. En esta misma línea, Berrio, Angulo y Gil (2013c), expresan que:

Hoy día la sociedad actual viene estableciendo consenso en torno al nuevo paradigma del conocimiento. Las universidades no escapan ante esta realidad, su reto fundamental consiste en enfrentar cambios, desarrollando nuevas herramientas de análisis, transformando mentalidades y actitudes para que se adapten a las necesidades emergentes basadas en la información y el conocimiento (pág. 123).

Es axiomático que para que el docente pueda desempeñar su praxis educativa eficazmente, es importante y una tarea casi imperiosa su inclusión en el medio investigativo, puesto que, el mundo cambia de forma exponencial, por tanto el docente debe evolucionar y actualizar su cumulo de conocimientos para estar en consonancia con las exigencias de la actualidad, aunado a esto, 
formar el individuo que tanto demanda la sociedad; igualmente trascender en la generación de estudios y proyectos productivos que contribuyan al progreso de la nación.

Partiendo de esta premisa, Palma (2010), considera que:

la gerencia del proceso investigativo en educación universitaria debe propiciar estrategias que generen políticas científicas integradoras dentro del contexto universitario enfocadas a enfrentar las demandas científico- técnicas de la sociedad y la economía en un futuro a corto, mediano y largo plazo; destaca que mediante una adecuada organización e integración de los grupos de investigación se puede motorizar y optimizar el impacto de las investigaciones que se ejecutan (pág. 3).

Posteriormente, Figueredo (2017): destaca que no existe saber único que proporcione todos los rasgos que despliega la gerencia y que emplean los gerentes; argumenta además que "...la gerencia tanto en su discurso teorizante como en su práctica cotidiana, es una especie de crisol donde se forja un conocimiento puesto al servicio del colectivo y a la permanente búsqueda del bienestar humano como propósitos cimeros" (pág. 330).

\section{Reflexiones finales}

Las universidades deben apuntar hacia la generación de investigaciones transcendentales, que consideren la axiología como premisa primordial, con una visión holística del ser, donde se comprenda que el hombre no es un ser aislado.

Al mismo tiempo, iniciando con investigaciones capaces de generar soluciones en problemas sociales básicos, aumentando gradualmente la complejidad de las mismas, con un constante monitoreo de sus procesos aplicando reingeniería de los mismos, para construir un sistema vivo en constante evolución, donde existan políticas que permitan estandarizar procesos investigativos comunes, que conlleven al máximo aprovechamiento 
del tiempo y los recursos dejando poco espacio para la repetición de ideas y por el contrario se puedan nutrir o actualizar investigaciones anteriores complementándose entre si; sin barreras de campos $u$ áreas del saber en un esquema de avance sostenido conveniente para toda la comunidad universitaria.

Asimismo, interpretando a Morin, incorporar una visión compleja, capaz de articular y enlazar criterios que se rechazan entre sí, siendo desglosados en apartados herméticos por falta de esta. Con esto no impulsa apartar lo simple, sino se trata de observarlo y articularlo a otros.

Por consiguiente, las universidades están llamadas a ser indiscutiblemente los puntos de crecimiento, en un cuadro histórico en donde adquiere suma importancia la capacidad para producir y aplicar nuevos conocimientos, los cuales deben desencadenar una indudable calidad de vida para todos, razonablemente esta generación de conocimientos y saberes se obtiene principalmente a través de la investigación universitaria.

El reto de la Universidad Politécnica Territorial Manuela Sáenz, radica en la forma como los gerentes deben enfocar sus prácticas gerenciales a través de un liderazgo oportuno y efectivo, en este sentido transformacional desde un enfoque complejo, que le permita como indica Morin transformar el pensamiento de manera que se traduzca a uno con visión amplia hacia nuevos horizontes; no uno que separe y mutile la realidad.

Para esto, la Institución de educación universitaria, demanda gestión del conocimiento, planificación y desarrollo, además de implementar y establecer un sistema que le permita aprovechar los conocimientos explícitos (documentados), así; como los tácitos del (individuo) que están alojados dentro de los miembros que conforman la universidad; de manera que estos conocimientos sean trasmitidos, argumentados y retroalimentados entre todos los investigadores desde diversas área del saber, para así facilitar la creatividad, donde surja la innovación continua logrando que se gestione 
efectivamente el conocimiento en investigación dentro de esta universidad desde la visión compleja.

\section{Referencias}

Balza, A. (2009). Pensar la Investigación Postdoctoral desde una Perspectiva Transcompleja. Investigación y postgrado, 24(3), 45-66, ISSN: 1316-0087. Recuperado de:

https://dialnet.unirioja.es/servlet/articulo?codigo $=3674413$

Barroso, F. (2011). Gestión del Conocimiento en Instituciones de Educación Superior y Centros de Investigación Científica en el Estado de Yucatán. Área de Investigación: Formación Profesional en Contaduría, Administración e Informática. México: Ciudad Universitaria. Recuperado de:

http://congreso.investiga.fca.unam.mx/docs/xvi/docs/9E.pdf

Berrio, H., Angulo, F., \& Gil, I. (2013a,b,c). Gestión del conocimiento como bases para la Gerencia de Centros de Investigación en Universidades Públicas. Dimensión Empresarial, 11(1), 116-125.

Recuperado de: http://dx.doi.org/10.15665/rde.v11i1.165

Fernández, C., \& Quintero, N. (2017). Liderazgo Transformacional y Transaccional en emprendedores venezolanos. Revista Venezolana de Gerencia, 22(77), 56-74, ISSN: 1315-9984. Recuperado de: http://www.redalyc.org/articulo.oa?id=29051457005

Figueredo, C. (2017). La Gerencia Avanzada en el Proceso de Investigación Científica Universitaria. Revista Scientific, 2(6), 322336. Recuperado de: https://doi.org/10.29394/scientific.issn.25422987.2017.2.6.17.322-336

Gaceta Oficial de la República Bolivariana de Venezuela (2010). Decreto No. 7.570, mediante el cual se crea la Universidad Politécnica Territorial del Norte del Táchira Manuela Sáenz, en el marco de la 
Misión Alma Mater, como Universidad Nacional Experimental. Gaceta Oficial N 5.987 Extraordinario, de fecha viernes 16 de julio. Caracas, Venezuela: La Asamblea Nacional de la República bolivariana de Venezuela. Recuperado de:

https://www.urbe.edu/UDWLibrary/GacetasAdvance.do?operator=EMP TY\&word=RESPONSABILIDAD\%20SOCIAL\%20EMPRESARIAL\&tag =TODO\&index $=1$

García, B. (2016). Gestión del Conocimiento desde el Contexto Universitario. Red Peruana de Divulgadores Científicos. IBERCIENCIA. Comunidad de Educadores para la Cultura Científica. Perú: Universidad Nacional de Tumbes. Recuperado de:

https://www.oei.es/historico/divulgacioncientifica/?Gestion-delconocimiento-desde-el-contexto-universitario

Gil, J., Domínguez, R., Garcia, L., Gándara, J., \& Mathison, L. (2012). La Investigación Universitaria como Eje de la Transferencia Social del Conocimiento. Publicaciones en ciencias y Tecnologías, 6(1), 41-51, ISSN: 1856-8890. Recuperado de: http://bibvirtual.ucla.edu.ve/cgiwin/be alex.exe?Acceso=T070200000931/4\&Nombrebd=PCYT\&Tipo Doc $=$ S\&Sesion $=131095$

León, R., Tejada, E., \& Yataco, M. (2003). Las Organizaciones Inteligentes. Industrial Data, 6(2), 82-87, ISSN: 1560-9146. Recuperado de: http://www.redalyc.org/articulo.oa?id=81660213

Marín, L. (2010). Gestión del Conocimiento y Cultura Investigativa. Una Aproximación Teórica-Critica. Praxis, [S.I.], 53-74, ISSN: 2389-7856. Recuperado de:

http://revistas.unimagdalena.edu.co/index.php/praxis/article/view/79

Osorio, S. (2012a,b). El pensamiento complejo y la transdisciplinariedad fenómenos emergentes de una nueva racionalidad. Revista Facultad de Ciencias Económicas, 20(1), 269-291, ISSN impreso: 0121- 
6805, ISSN electrónico: 1909-7719. Recuperado de:

https://doi.org/10.18359/rfce.2196

Palma, M. (2010). Espacio Iberoamericano del Conocimiento. Gestión del

Conocimiento desde la Investigación Educativa. Buenos Aires, República Argentina: Congreso Iberoamericano de Educación, METAS 2021. Recuperado de:

http://webmail.adeepra.com.ar/congresos/Congreso\%20IBEROAMERI CANO/EIC/R1758 Poster Palma.pdf

Pereira, J. (2010). Consideraciones Básicas del Pensamiento Complejo de Edgar Morín, en la Educación. Revista electrónica Educare, XIV(1), 67-75. ISSN: 1409-4258. Recuperado de:

http://www.redalyc.org/articulo.oa?id=194114419007

Pérez, L., \& Alfonso, N. (2016). Conocimiento, Educación y Transcomplejidad. Educere, 20(65), 11-20, ISSN: 1316-4910. Recuperado de: http://www.redalyc.org/pdf/356/35646429002.pdf

Ramos, A., \& Isa, G. (2010). La Cultura Investigativa Transcompleja en las Universidades sobre las Personas con Discapacidad. Blogger.com: Epistemología de la Ciencia. [Documento en línea]. Recuperado de: http://epistemologiauba.blogspot.com/2010/12/la-cultura-investigativatranscompleja.html 


\section{Ingrid Yorley Guerrero Rivera}

e-mail: teacher272777@gmail.com

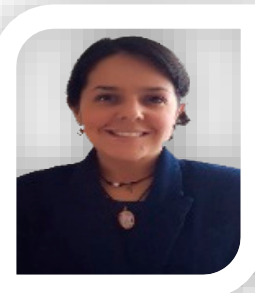

Nacida en Venezuela. Licenciada en Educación Mención Inglés, otorgado por la Universidad de los Andes, Táchira (ULA), MSc. en Gerencia Educativa, otorgado por la Universidad Experimental del Táchira (UNET), con mención publicación y honorifica. Actualmente, Docente Universitaria, agregada a Dedicación Exclusiva de la Universidad Politécnica Territorial del Norte del Táchira "Manuela Sáenz". Tutora de diversos Proyectos Socio Tecnológicos en la Institución en la que laboro en los distintos PNF. Línea de investigación: La Gerencia en la Sociedad de la Información. 